\title{
A Pharmacist's Role in a Dental Clinic: Establishing a Collaborative and Interprofessional Education Site
}

Kalin L. Johnson, PharmD ${ }^{1,2}$; Kevin T. Fuji, PharmD, $M A^{1,3}$; Joseph V. Franco, DDS ${ }^{2}$; Shana Castillo, Pharm $D^{1}$; Karen $O^{\prime}$ Brien, Pharm $D^{1}$; Kimberley J. Begley, PharmD ${ }^{1}$

${ }^{1}$ Creighton University School of Pharmacy and Health Professions, Omaha, Nebraska

${ }^{2}$ Creighton University School of Dentistry, Omaha, Nebraska

${ }^{3}$ Creighton University Center for Health Services Research and Patient Safety, Omaha, Nebraska

\begin{abstract}
Background: Dental patients often have comorbidities and take multiple medications, some of which could impact their dental health and treatment. A pharmacist in a dental clinic can assist with the gathering, documentation and evaluation of a dental patient's medication history as it pertains to their dental visit and overall health.

Purpose: To develop and implement a collaborative and interprofessional education program with a pharmacist providing services in a dental school clinic.

Summary: Creighton University School of Dentistry, a student-operated dental clinic located in Omaha, Nebraska, provides dental care by student dentists, faculty and staff to the surrounding community in a learning-focused environment. A pharmacist was incorporated into the dental clinic to create and establish an interprofessional relationship with both dental students and faculty beginning August 2014. Pharmacy students on an ambulatory care advanced pharmacy practice experience rotation were eventually added to the team. The pharmacy team provided medication therapy management services including disease state and medication counseling, medication reconciliation, identifying drug-related problems and dental implications of medications, and recommendations for prescribed medications.

Conclusion: The pharmacy team's presence was largely accepted by dental faculty, staff, dental students, and patients. Pharmacists can play an important role in a dental clinic by performing thorough health and medication histories and communicating with dental and medical providers involved in a patient's care.
\end{abstract}

Keywords: Pharmacist, Interprofessional, Dental, Interprofessional Education (IPE), Dental Clinic

\section{Background}

Due to its routine nature, a dental visit can serve as the first line of defense for early detection, treatment, and prevention of both oral and systemic diseases. ${ }^{1}$ Dentists regularly encounter patients whose current medications may influence dental treatment, including the choice of prescribed medications. ${ }^{2}$ This is particularly a problem with elderly patients, and as the number of elderly patients in the United States grows (one in every five U.S. residents will be over age 65 by 2030 according to the U.S. Census Bureau), so does the prevalence of chronic conditions and medication use.,4 For example, xerostomia, tooth loss, edentulism, and periodontal disease are common dental conditions amongst the elderly population and are often due to, or worsened by, patients' medication regimen. ${ }^{2}$ Due to their potential impact on patient care, all medication regimens should be documented and evaluated by dentists. Best practice dictates dentists obtain a thorough medication history and evaluate the potential for drug-drug or drug-disease interactions, adverse effects on the oral cavity and related structures, medication dependence, and antibiotic resistance. ${ }^{5}$ Drug interactions commonly encountered by dental

Corresponding author: Kalin L. Johnson, PharmD

Creighton University SPAHP

2500 California Plaza, Omaha, NE 68178

Email: kalinjohnson@creighton.edu professionals include nonsteroidal anti-inflammatories with antihypertensives, antidepressants with sedatives, sedatives with central nervous system depressants, vasoconstrictors with nonselective $\beta$-adrenergic blocking drugs, antibiotics with cytochrome P450 enzyme inducers or inhibitors, and antiplatelet, analgesic, and anticoagulant medications with dental treatment. ${ }^{6-11}$ However, dental training typically does not include an intensive focus on pharmacology and/or pharmacotherapeutics that would allow dentists to comprehensively identify and address these types of interactions. As the medication use experts, pharmacists are well equipped to assist dentists with the challenges discussed above.

A 2015 study determined that pharmacist-compiled medication histories identified medication omissions, therapeutic duplications, and/or errors in dosages and directions in nearly $90 \%$ of medication records in dental profiles. ${ }^{12}$ Compared to non-pharmacist healthcare providers, it is well-established that pharmacists obtain higher quality medication histories with fewer errors and a more complete listing of all medications used by patients. ${ }^{13-20}$ Additionally, pharmacists in the dental clinic setting can identify potential interactions and adverse effects, provide dentists and staff with updates on evidencebased guidelines, recommend self-care products that promote good oral health, and provide medication therapy management (MTM) services. ${ }^{5,21}$ For interprofessional interactions to occur 
in practice, it is vital that students engage in interprofessional education (IPE) to improve health outcomes and learn from each other during their time in school. ${ }^{22}$

Despite the identified benefits of pharmacist-dentist interprofessional collaboration, a review of the literature illustrates that routine collaboration between the professions is lacking. 1,5,12,20,23-28. Thus far there have only been six studies published in this area. Four studies found that a pharmacydental collaboration (two occurring in a free dental care clinic and two in a university dental clinic setting), yielded a greater reduction in medication history omissions, medication discrepancies, decreased opioid prescribing overall, and greater patient perceived knowledge of and intentions to utilize medications to assist with tobacco cessation when compared to patients not benefiting from interprofessional care. ${ }^{12,20,25,26} \mathrm{~A}$ fifth and sixth study described two different IPE models between dentists and pharmacists in a university dental clinic setting. ${ }^{27,28}$ The interprofessional team in the study by BranchMays et. al., assisted in identifying drug therapy problems and acquiring primary care metrics (diabetes status, tobacco use, blood pressure and pulse), a need that is rarely met in dental clinics. ${ }^{27}$ The study by Pogge et. al., discovered that when pharmacy and dental students were integrated in a dental clinic, both pharmacy and dental students' attitudes and knowledge about IPE were improved. ${ }^{28}$ This early published evidence indicates that collaborative care in educational and clinical dental settings may result in better mutual understanding of the role of the pharmacist and dentist, a strengthened knowledge base of pharmacotherapy as it applies to dentistry, and, ultimately, improved patient outcomes. ${ }^{5,12,20 \text {, }}$ 25-28 Thus, there is a need to identify and outline the development of structured and sustainable pharmacy-dental collaborative practice models.

The purpose of this paper is to describe the development of a formal interprofessional care team consisting of dentists, dental students, a pharmacist, and pharmacy students in a university dental school clinic.

\section{Clinic Operation}

This was a joint initiative by the Creighton University School of Dentistry and School of Pharmacy and Health Professions. The primary goals of this interprofessional care team were to: 1 ) provide MTM and other pharmacy-related services to enhance the outcomes of dental clinic patients; and 2) provide opportunities for interprofessional collaborative experiences between pharmacy and dental students.

This pilot effort took place in a dental clinic operated by the Creighton University School of Dentistry in Omaha, Nebraska. The clinic provides quality and affordable dental care to primarily low-income, elderly, and uninsured (62.9\%) or Medicaid patients. Most patients reside in Nebraska (80.7\%) and lowa (17.1\%) but $1.9 \%$ of patients travel to the clinic from 34 other states $(0.02 \%$ of patients had undocumented residence). Patient demographics (current as of July 2018) are provided in Table 1 for all active patient profiles. Active patients are individuals who have been seen at least once in the previous two years. The overall impact of this clinic is large, serving over 11,000 patients annually. ${ }^{29}$ As shown in Table 1, $58.78 \%$ of the patient population is over 39 years of age and $37.6 \%$ is over the age of 60 . As the elderly are more likely to have multiple comorbidities and medications, the potential impact of pharmacy services in the clinic was deemed high. ${ }^{2-4}$ The clinic is open 28 hours per week and offers a wide range of dental and specialty services including assessment, general dentistry, fixed prosthodontics, periodontics, endodontics, oral surgery, acute care, and oral diagnostics. Treatment is performed by third- and fourth-year dental students (approximately 172 students), as part of the four-year dental curriculum, working under the supervision of licensed dentists.

The main area of the dental clinic occupies one large room, with each patient seated in a private cubicle with chairs, a computer, and dental supplies. The clinic employs a team of full-time and part-time dental faculty, hygienists, and support staff. All thirdand fourth-year dental students attend assigned dental clinic sessions, with 129 cubicles available for patient appointments. Clinic sessions operate in four-hour blocks, and the complexity and comprehensive needs of the patients often result in one patient occupying a chair throughout the full four hours. The extended dental appointments allow students ample time to complete their work while supervised by faculty who approve all treatments and recommendations and refer patients to other specialty departments of the clinic. In addition to the main area, the dental clinic also houses an Assessment Clinic and Oral Surgery department.

The pharmacist began work in the clinic in August 2014, with initial efforts directed toward Oral Diagnostics and Oral Surgery from August 2014 to July 2016, and expansion to the Assessment Clinic in August 2016. In August 2015, the pharmacist integrated fourth-year Doctor of Pharmacy students completing their ambulatory care Advanced Pharmacy Practice Experience (APPE). The combination of pharmacist and fourth-year pharmacy students constituted the pharmacy team. The pharmacist is present in the clinic four days a week but is available by consultation request five days a week. In addition, two fourth-year pharmacy students are typically onsite from mid-August through April. The responsibilities of the pharmacy team and collaboration with dentists and dental students in the context of the clinic workflow is described.

\section{Dental Assessment Clinic}

Patients begin with a short visit to the Assessment Clinic, which operates with four cubicles and serves approximately 26 patients each clinic session. An initial 15-minute evaluation is conducted to determine if the patient can safely receive treatment or if health conditions are present that would preclude care (e.g., uncontrolled diabetes and hypertension, active seizure disorder, severe cardiac disease, severe 
psychiatric disease, etc.). During this evaluation, the pharmacy team obtains a medication history, performs medication reconciliation, and assesses medication adherence. The pharmacy team uses this information to assist the dental team with assessing the patient's readiness for dental treatment and making physician referrals for uncontrolled medical conditions and/or medication adherence issues. Once patients complete their visit to the Assessment Clinic, they move on to a lengthier appointment in Oral Diagnostics for an oral evaluation and medical review including consults from other specialty departments.

\section{Oral Diagnostics}

Oral Diagnostics operates with the largest number of cubicles (22) and sees the largest number of patients. The majority of the pharmacy team's time and effort are focused in this department. During the first hour of the four-hour appointment, the pharmacy team works independently using the dental clinic's electronic health record (EHR) system to evaluate and compile patient information. This includes assessing dental students' documentation for accuracy and completeness of a patient's health history (including a recorded blood pressure and most recent hemoglobin A1c and blood glucose values if the patient has diabetes mellitus) and medication history (name of each medication, dosage, frequency, indication, and dental adverse events). If information is incorrect or missing, the pharmacy team communicates the issue to the assigned dental student and assists with obtaining and documenting pertinent information.

Remaining clinic time is spent entering and documenting interventions in the EHR and counseling patients. A checklist was developed to document all pharmacy team interventions. Interventions were categorized into six domains: adverse reactions and/or drug interactions, drug selection problems, medication dosing problems, patient-related problems, medication history assistance, and dental medication recommendations. These domains were identified through consultation with faculty experts in ambulatory pharmacy care. Definitions of intervention categories can be viewed in Table 2 . If problems are identified, they are addressed with the appropriate party (patient, primary care provider, dentist, dental student etc.) and both the problem and intervention are documented using the checklist. Primary care providers are notified of a medication or health condition issue via an electronic request for medication change form faxed from the $E H R$, or a call made to the primary care provider's office. If a patient has three or more comorbid conditions and five or more medications, the pharmacy team also offers a comprehensive medication review and screening for other intervention opportunities such as smoking cessation, lifestyle modifications, or disease state counseling. These activities are completed with the dental student present to facilitate collaboration and efficient exchange of information with the pharmacy team.

\section{Oral Surgery}

Oral Surgery operates with one cubicle for oral surgery evaluation and seven cubicles for procedures. The two major responsibilities of the pharmacy team are rounding with the dental team to discuss and resolve medication-related problems prior to procedures and, by consultation request, to answer questions, review medication histories and counsel patients on prescribed medications.

\section{Establishing Services}

To establish this interprofessional collaboration, the pharmacist was given a secondary faculty appointment in the School of Dentistry and tasked with leading the development of a formal interprofessional practice and education site in the university's dental school clinic. Given the increasing focus on collaborative practice models nationally and the requirement of both pharmacy and dental accreditation standards for students to participate on an interprofessional care team, the Interprofessional Education Collaborative core competencies were used to guide the development of this collaboration. ${ }^{30}$

To identify the needs of the clinic and areas of opportunity, the pharmacist gathered information from dental faculty and students through attendance at faculty and staff meetings, small group discussions, informal conversations, patient charts, and shadowing. All dental students, faculty, and staff were notified via email about the pharmacy team's role in the clinic. Engaging stakeholders early in the interprofessional collaborative process was crucial to obtain buy-in, establish trust, and ensure the pharmacy team was appropriately placed within the dental clinic workflow.

Initially, the pharmacist was available for consultation in all areas of the clinic. However, with numerous dental cubicles operating simultaneously and only one pharmacist, the patient cases chosen for pharmacist intervention required thoughtful selection. The Assessment Clinic, Oral Diagnostics, and Oral Surgery were selected to maximize the impact of pharmacy team interventions by reaching patients early on in their dental treatment and targeting patients with the greatest potential to experience drug interactions.

\section{Evaluation}

The Institutional Review Board approved the tracking and reporting of pharmacist intervention data from August 2014 to July 2018 as "not human subject research" and, therefore, a review was not required. In addition, the name of each collaborating dental student was recorded to ensure that all students engaged in interprofessional activities. The collected data is displayed in Table 2.

During the tracking period, the pharmacy team reviewed charts for 6,596 patients. Assistance with medication history (obtaining, documenting, reconciling, and correcting) was provided for 4,335 patients $(65.7 \%)$, and interventions were made with 2,438 patients $(36.9 \%)$. As more than one 
intervention could be made for a patient, a total of 2,773 interventions were made and documented: adverse reaction/drug interaction problem $(n=1,206)$; drug choice problem ( $n=778)$; patient-related problem $(n=757)$; and dosing problem $(n=321)$. In addition to the data described in Table 2 , the pharmacy team impacted drug-choice prescribing of dental medications in another 142 patients by helping dentists select the most appropriate antibiotic and analgesic medications. Patient counseling was provided to address common patientrelated problems, such as adherence, insufficient awareness of disease state, or problems affording medication.

\section{Challenges}

The pharmacy team encountered several challenges in establishing services and performing patient care duties. Many of these are unique to a dental clinic setting.

\section{Acceptance of the Interprofessional Role}

There was an on-going challenge of getting various health professionals to embrace the importance and positive outcomes related to interprofessional collaborative practice. Dentists have long been excluded from the interprofessional conversation. Permanently integrating a member of another health profession into their workflow and clinical space was a new experience for the dental team. Most dental faculty, staff and students welcomed the medication-related assistance but struggled with, and at times pushed back against, perceived pharmacy-related "interruptions." Additionally, the pharmacy team often called physicians' offices and were occasionally questioned about why pharmacists in a dental clinic needed information such as patients' medication histories or hemoglobin A1C values for patients with diabetes mellitus. Even some fourth-year pharmacy students struggled with the idea of including dentists on an interprofessional team and were uncomfortable presenting a medication analysis to their dental colleagues. Ongoing discussions with students revealed a lack of knowledge and understanding about a pharmacist's potential contributions to optimizing dental care. Finally, dental clinic patients are unaccustomed to being approached by a pharmacist to gather medication histories and assess adherence to prescribed therapies, resulting in some patients refusing to engage with the pharmacy team. Anyone wishing to replicate this model should place a priority on educating other members of the healthcare team about the role of the pharmacy team and how this interprofessional collaboration facilitates better patient care.

\section{Physical Space}

Due to the prior organization and space constraints of the dental clinic building, there was inadequate infrastructure to accommodate a dedicated pharmacy counseling space or pharmacy-specific computers. The pharmacist had to identify and advocate for the resources and space needed to adequately support the pharmacy team's collaborative role. Schools or colleges wishing to incorporate a similar collaborative program should proactively identify space, technology, and personnel needs, and ensure that these resources are in place prior to initiation of the collaborative practice efforts.

\section{Access to Patients}

Although patients are often in the clinic for four-hour appointments, they are not available to the pharmacy team during a large portion of that time. They may be unable to speak due to dental treatment and evaluations, in radiology, or in other clinic specialty areas for consultations. With a small pharmacy team, it was sometimes difficult to find a counseling window that did not interfere with dental treatment. This was especially true in Oral Surgery where the window to discuss medications falls within the first 30 minutes of the appointment, and all patient appointments commonly started at the same time. Pharmacy teams should anticipate this need to adapt workflow to ensure patients receive the pharmacy services needed to optimize the safety and efficacy of dental treatment.

\section{Preliminary Outcomes}

This is an ongoing early effort and as such, quantifiable outcomes are not yet available. Anecdotally, this collaboration was a success, aided by significant communication and partnership between the pharmacy and dental teams. With support and encouragement from the pharmacist and dentists, students learned the importance of acquiring a complete medication history and being fully informed about each of their patient's medications, even when they did not have an obvious dental adverse effect or implication. Dental students quickly observed how helpful the pharmacy team could be and routinely utilized the team for consults. The dental team proactively informed patients to expect a visit from the pharmacy team. The willing cooperation of most patients was likely attributable to the dental team's strong support of the interprofessional collaboration effort.

An ongoing limitation of the pharmacy team's intervention data is a lack of knowledge regarding the outcomes of some interventions. While outcomes are actively being collected for medication recommendations within the clinic, issues brought to the attention of a patient's primary care provider, referrals, or counseling to improve patient knowledge/compliance are not tracked. Occasionally, the team saw patients during followup clinic visits or received written responses from primary care providers, but typically, patients did not return for follow-up appointments, or the results of medication recommendations were not known. Many patients served by the dental clinic do not receive routine health or dental care, are of low socioeconomic status, are recent immigrants to the country, come from out of state or spend a majority of the year living out of state, change addresses frequently or have no permanent address, do not have telephones, or have other unique circumstances. Thus, the clinical impact of the pharmacy team on patient-related outcomes is difficult to assess. However, given the fact that this patient population 
would likely not otherwise receive these services, the pharmacy team interventions are still of value despite the current lack of knowledge about comprehensive impact on clinical outcomes.

\section{Future Plans}

The specialty practices selected by the pharmacy team as focus areas have shifted over time and through lessons learned. Initially, the pharmacy team planned to divide their time equally between Oral Diagnostics and Oral Surgery. Because medications are used and frequently prescribed in Oral Surgery, it was believed that integrating the pharmacy team into this specialty area would be useful. However, the oral surgeons already had detailed knowledge about medications and their impact on treatment decisions and did not require regular support from the pharmacy team. Ongoing meetings with the dentists identified a need for pharmacy assistance in the Assessment Clinic, and this area has continued to provide consistent opportunities for pharmacy consultations and interventions. Through collaborative planning sessions, the pharmacy and dental teams will continue to assess which specialty practices would benefit most from pharmacy support. Periodontology will likely be the next area for expansion of services given the established connection between periodontal disease and uncontrolled diabetes mellitus. ${ }^{31}$

Notifying the dental students, faculty, and staff electronically about the integration of the pharmacy team into the clinic was an efficient means for disseminating information quickly. However, in retrospect, notifying all stakeholders in-person would have been more effective. Potential mechanisms include introduction of the pharmacist and explanation of the role of the pharmacy team during staff meetings and in the classroom. Such a process would allow time for a discussion of the goals of interprofessional collaboration between pharmacy and dental teams, as well as opportunities to answer any stakeholder questions. The pharmacist is working with dental faculty to identify a time for the pharmacist to describe the pharmacy team's objectives, role, expectations, and importance of acquiring an accurate and complete medication history.

Although the pharmacist maintained records of the interventions completed, the dental EHR lacked a specific pharmacy consultation form. The pharmacist has been working with the dental school's information technology department to develop a pharmacy consultation form that would allow the pharmacist to separately document all pharmacy-related interventions. This form will remain attached to the patient's chart and will facilitate awareness of pharmacy team interventions among all members of the healthcare team. Additionally, this form can be used to help track pharmacy interventions and related clinical outcomes resulting from those interventions.

As described, the fourth-year pharmacy students who are part of the pharmacy team in the dental clinic are completing an APPE in ambulatory care. Pharmacy students can observe their dental colleagues evaluating patients and obtaining medication histories. They practice effective communication skills and problem solving with dental students, dentists, staff, and patients; counsel patients on new and current medications; educate dental students about important patient-specific medication information; and learn how to review a patient's medication and health history from the perspective of an interprofessional team member. Offering this APPE program in a dental setting provides a unique opportunity for interprofessional interactions and warrants its own evaluation of effectiveness. The authors intend to develop a separate paper specifically describing these efforts and the resulting student learning outcomes.

\section{Conclusion}

An interprofessional education site was successfully developed and implemented in a university dental clinic through the collaborative efforts of dentists and a pharmacist. Integrating the pharmacy team into clinic operations provided dentists and dental students with medication-related support and contributed to more robust dental and overall healthcare for patients. The pharmacy team plans to alter/expand their patient population focus, intervention tracking, and involvement in pharmacy and dental school curricula to address challenges that arose in implementing this program. Additional research will be conducted to determine this program's impact on patient outcomes, as well as attitudes and abilities of pharmacy and dental students relative to participating on an interprofessional team.

Acknowledgements: The authors would like to thank John Shaner, D.D.S. and Jason Goodchild, D.D.S. of Creighton University's School of Dentistry for their promotion of interprofessional education.

\section{Funding/Support: None}

\section{Conflicts of Interest: None}

\section{References}

1. Wilder RS, O'Donnell JA, Barry M, et al. Is dentistry at risk? A case for interprofessional education. J Dent Educ. 2008;72(11):1231-1237.

2. Weinstock R, Johnson M. Review of Top 10 Prescribed Drugs and Their Interaction with Dental Treatment. Dent Clin N Am. 2016;60:421-434.

3. Moore PA, Gage TW, Hersh EV, et al. Adverse drug interactions in dental practice: professional and educational implications. JADA. 1999:130;47-54.

4. United States Census Bureau. (2018, March 13). Older people projected to outnumber children for first time in U.S. history. https://www.census.gov/newsroom/pressreleases/2018/cb18-41-population-projections.html. 
5. Lygre H, Kjome RLS, Choi H, Stewart AL. Dental providers and pharmacists: a call for enhanced interprofessional collaboration. Int Dent J. Available at: http://onlinelibrary.wiley.com/doi/10.1111/idj.12304/fu II. Accessed July 26, 2017.

6. Salort-Llorca C, Mínguez-Serra MP, Silvestre-Donat FJ. Interactions between ibuprofen and antihypertensive drugs: Incidence and clinical relevance in dental practice. Med Oral Patol Oral Cir Bucal. 2008;13(11):E717-721.

7. Lambrecht JT, Greuter C, Surber C. Antidepressants relevant to oral and maxillofacial surgical practice. Annals of Maxillofacial Surgery. 2013;3(2):160-166.

8. Hersh EV. Adverse Drug Interactions in Dental Practice: Interactions involving antibiotics. Part II of a series. JADA. 1999;130:236-251.

9. Hersh EV, Moore PA. Drug interactions in dentistry: The importance of knowing your CYPs. JADA. 2004;135:298311.

10. Moore PA. Adverse Drug Interactions in Dental Practice: Interactions associated with local anesthetics, sedatives and anxiolytics. Part IV of a series. JADA. 1999;130:541-554.

11. Yagiela JA. Adverse Drug Interactions in Dental Practice: Interactions associated with vasoconstrictors. Part $\mathrm{V}$ of a series. JADA. 1999;130:701-709.

12. Choi H, Stewart A, Rosenfeld B, et al. Medication discrepancies in the dental record: implications on oral health. J Am Pharm Assoc. 2015;55:e169.

13. Mekonnen, AB, McLachlan AJ, Brien JE. Pharmacy-led medication reconciliation programmes at hospital transitions: a systematic review and meta-analysis. J Clin Pharm Ther. 2016;41:128-144.

14. Reeder TA, Mutnick A. Pharmacist-versus physicianobtained medication histories. Am J Health-Syst Pharm. 2008;65:857-860.

15. Steurbaut $S$, Leemans $L$, Leysen $T$, et al. Medication History Reconciliation by Clinical Pharmacists in Elderly Inpatients Admitted from Home or a Nursing Home. Ann Pharmacother. 2010;44:1596-1603.

16. Stewart AL, Lynch KJ. Medication discrepancies despite pharmacist led medication reconciliation: the challenges of maintaining an accurate medication list in primary care. Pharmacy Practice. 2014;12(1):360.

17. Hatch J, Becker T, Fish J. Difference between pharmacist-obtained and physician-obtained medication histories in the intensive care unit. Hosp Pharm. 2011;46(4):262-268.

18. de Andrés-Láaro AM, Sevilla-Sánchez D, del Mar OrtegaRomero, $\mathrm{M}$, et al. Accuracy in the medication history and reconciliation errors in the emergency department. Med Clin (Barc). 2015;145(7):288-293.

19. Splawski J, Minger $\mathrm{H}$. Value of the Pharmacist in the Medication Reconciliation Process. Pharmacy and Therapeutics. 2016;41(3):176-178.
20. Rotz ME, von Vital R, Radovanovich A, et al. Impact of an interprofessional practice experience on medication histories within a dental admissions clinic. Journal of Interprofessional Education \& Practice. 2018;10:15-23.

21. Medication therapy management in pharmacy practice: core elements of an MTM service model. Version 2.0. The American Pharmacists Association and the National Association of Chain Drug Stores Foundation (2008). Available at: https://www.pharmacist.com/sites/default/files/files/co re elements of an $\mathrm{mtm}$ practice.pdf. Accessed July 26, 2017.

22. Framework for action on interprofessional education \& collaborative practice. Geneva: World Health Organization. World Health Organization (WHO). (2010). Available at: http://apps.who.int/iris/bitstream/10665/70185/1/WH O HRH HPN 10.3 eng.pdf?ua=1. Accessed July 26, 2017.

23. Conway SE, Smith WJ, Truong TH, Shadid J. Interprofessional pharmacy observation activity for third-year dental students. J Dent Educ. 2014;78(9):1313-1318.

24. Wilhelm M, Poirier T, Otsuka A, Wagner S. Interprofessional ethics learning between schools of pharmacy and dental medicine. J Interprof Care. 2014;28(5):478-480.

25. Stewart A, Zborovancik KJ, Stiely KL. The impact of pharmacy services on opioid prescribing in dental practice. J Am Pharm Assoc. 2017;57:578-582.

26. Virtue SM, Rotz ME, Boyd M, et al. Impact of a novel interprofessional dental and pharmacy student tobacco cessation education programme on dental patient outcomes. J Inter Prof Care. 2018;32(1):52-62.

27. Branch-Mays GL, Pittenger AL, Williamson K, et al. An interprofessional education and collaborative practice model for dentistry and pharmacy. J Dent Educ. 2017;81(12):1413-1420.

28. Pogge EK, Hunt RJ, Patton LR, et al. A pilot study on an interprofessional course involving pharmacy and dental students in a dental clinic. AM J Pharm Educ. 2018;82(3):217-223.

29. Creighton University School of Dentistry. Patients: Quality, Affordable Dental Care.

https://dentistry.creighton.edu/patients. Accessed July 24, 2017.

30. Interprofessional Education Collaborative. Core competencies for interprofessional collaborative practice: 2016 update. Washington, DC: Interprofessional Education Collaborative. 2016:1-19.

31. Daniel R, Gokulanathan S, Shanmugasundaram N, et al. Diabetes and periodontal disease. J Pharm Bioallied Sci. 2012;4(2):S280-S282. 
Table 1. Patient Demographics - Active patient profiles as of July 15, 2018

Total Active Patients: 14,889

\begin{tabular}{|l|l|l|}
\hline \multicolumn{1}{|c|}{ Gender } & \multicolumn{1}{|c|}{ Number of Patients } & \multicolumn{1}{c|}{ Percentage } \\
\hline Female & 7,773 & $52.21 \%$ \\
\hline Male & 7,114 & $47.78 \%$ \\
\hline Transgender & 2 & $0.01 \%$ \\
\hline \multicolumn{1}{|c|}{ Third Party Dental Coverage } & & \\
\hline No & 9,370 & $62.93 \%$ \\
\hline Yes State of Residence & 5,519 & $38.00 \%$ \\
\hline \multicolumn{1}{|c|}{ Age } & & \\
\hline Nebraska & 12,022 & $80.74 \%$ \\
\hline lowa & 2,549 & $17.12 \%$ \\
\hline Other States (34 total) & 286 & $1.92 \%$ \\
\hline None Documented & 31 & $0.02 \%$ \\
\hline & & \\
\hline$\leq 15$ & 2,255 & $15.15 \%$ \\
\hline $16-25$ & 1,345 & $9.03 \%$ \\
\hline $26-39$ & 2,536 & $17.03 \%$ \\
\hline $40-59$ & 3,154 & $21.18 \%$ \\
\hline $60-74$ & 3,517 & $23.62 \%$ \\
\hline$\geq 75$ & 2,082 & $13.98 \%$ \\
\hline & & \\
\hline
\end{tabular}

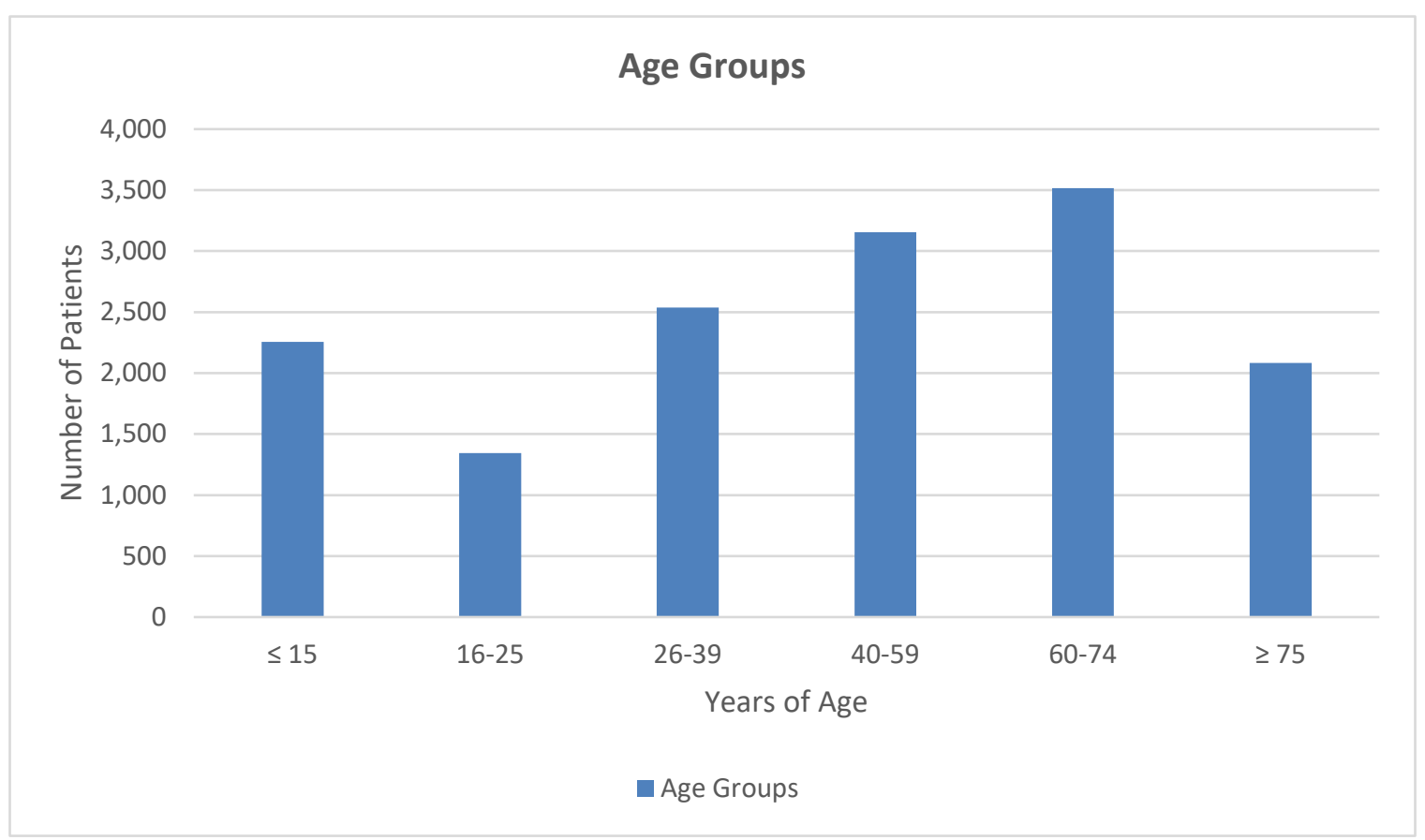


Table 2. Number and type of interventions made by the pharmacy team

\begin{tabular}{|c|c|c|}
\hline Type of Intervention & $\begin{array}{c}\text { Number of } \\
\text { Interventions }\end{array}$ & $\begin{array}{l}\text { Percentage of Charts } \\
\text { Reviewed } \\
\text { (n=6596) }\end{array}$ \\
\hline Adverse Reaction/Drug Interactions ${ }^{1}$ & 1206 & $18.28 \%$ \\
\hline Drug Choice Problems ${ }^{2}$ & 778 & $11.80 \%$ \\
\hline Patient-Related Problems ${ }^{3}$ & 757 & $11.48 \%$ \\
\hline Dosing Problems ${ }^{4}$ & 321 & $4.87 \%$ \\
\hline
\end{tabular}

${ }^{1}$ Evident side effect, allergic reaction, potential drug-drug, drug-food, drug-disease, or drug age interaction, deterioration of disease state, or new symptom/indication revealed

${ }^{2}$ Inappropriate drug/form/dosage/duplication, contraindication, no clear indication, or no drug but clear indication

${ }^{3}$ Patient dissatisfied with therapy, insufficient awareness of health and disease, questionable medication adherence, patient forgets to use/take medications, patient has concerns with medications, or patient unable to use medication/dosage form

${ }^{4}$ Drug not taken/administered, wrong drug taken/administered, drug dose too low/high, inappropriate timing of dosing, or duration of treatment too short/long 